\title{
Food Allergies
}

\author{
Contributor: , Margherita Di Costanzo ${ }^{1}$ \\ 1, Department of Pediatrics and Neonatology, Guglielmo da Saliceto Hospital, Piacenza, \\ Italy; Department of Translational Medical Science - Pediatric Section, University "Federico II", \\ Naples, Italy; ImmunoNutritionLab - CEINGE Advanced Biotechnologies, U; \\ M.DiCostanzo@ausl.pc.it
}

Version received: 28 July 2020

The increasing prevalence and severity of pediatric food allergies (FA) demands innovative preventive and therapeutic strategies. Emerging evidence suggests a pivotal role for the gut microbiome in modulating susceptibility to FA. Studies have demonstrated that alteration of gut microbiome could precede FA, and that particular microbial community structures early in life could influence also the disease course. The identification of gut microbiome features in pediatric FA patients is driving new prevention and treatment approaches.

\section{Introduction}

In the past two decades, the prevalence, persistence, and severity of food allergies (FA) have been increasing1]. This has led to an increased number of hospitalizations and costs for patients, their families, and healthcare systems ${ }^{[2]}$. Several hypotheses have been formulated to explain such phenomenon. Among these, the current "old friends and biodiversity hypotheses" propose that changes in living environment, diet, and lifestyle associated with Westernized countries have altered the microbial diversity, disrupting the immunoregulatory function of the microbiome and predisposing people to allergic sensitization[3][4][5]. The formulation of these hypotheses derives from robust evidence suggesting a key role of microbiome alteration, influenced by modern lifestyle factors, in the development of FA[6].

\section{Targeting Gut Microbiome in Food Allergy}

\subsection{Environmental Factors}

There are several modifiable environmental factors that can influence the occurrence of FA and can potentially be targeted to prevent FA. The window of opportunity, in which environmental factors determine an individual susceptibility to developing communicable and noncommunicable chronic diseases (including allergies) in adult life, is called the "first 1000 days". This period goes from intrauterine development to the first 2 years of life, during which gut microbiota and immune system development are strongly influenced by environmental factors ${ }^{[7]}$. Maternal diet during pregnancy and lactation exert a direct and indirect effect on maternal gut and mammalian gland microbiota (enteromammary pathway) and play a pivotal role in early influence on infant gut microbiome composition and function[8]. Other factors such as rural environment, vaginal delivery, increased family size, exposure to pets, breastfeeding, a high-fiber diet, and/or fermented food are associated with a protective effect against FA development. In contrast, cesarean section delivery, prenatal and early-life exposure to antibiotics, gastric acidity inhibitors, antiseptic agents, and junk-food-based and/or low-fiber/high-fat diets may increase the risk of FA development. These environmental factors are mostly related to the structure and function of the gut microbiome [9][10][11][12][13][14][15][16][17][18][19][20][21][22] (Figure 1). 


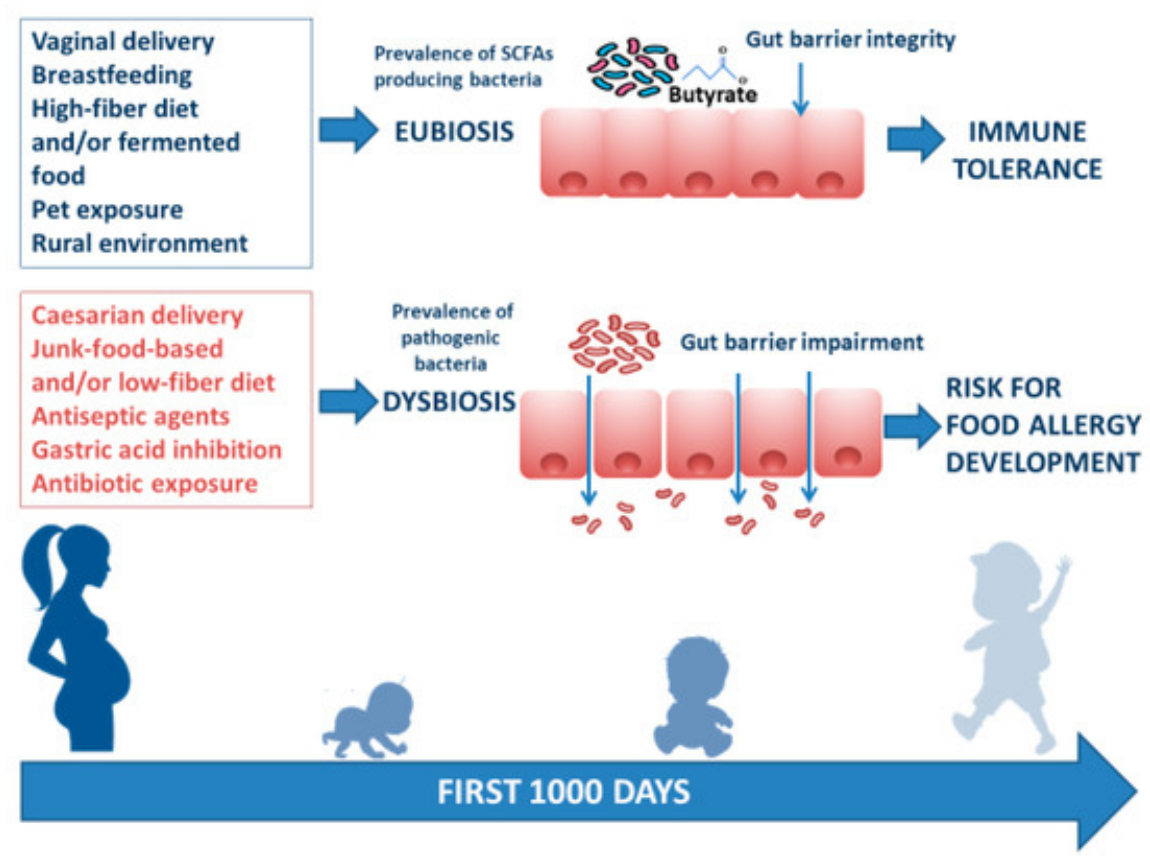

Figure 1. Infant gut microbiome composition and function is related to multiple environmental factors. The "first 1000 days" start from intrauterine development to the first 2 years of life and represent the frame of gut microbiome structure and function shaping. The ideal path begins with a full-term gestational period, followed by spontaneous delivery, breastfeeding provided by a mother following a Mediterranean diet lifestyle, earlier rural environmental exposure, and infant intake of a high-fiber diet and/or fermented food. All these factors are responsible for gut eubiosis, with a prevalence of SCFA-producing bacteria and gut barrier integrity, laying foundations for a healthy status and for a long-lasting protection against noncommunicable chronic diseases (such as FA) later in life. Conversely, caesarian delivery, from a mother following a junk-food-based and/or low-fiber diet, and direct or indirect childhood exposure to antiseptic agents and drugs (mainly antibiotics and gastric acidity inhibitors) leads to gut dysbiosis with prevalence of pathogenic bacteria, reduction of immunomodulatory factor production, increased gut barrier permeability, and a risk for FA development.

\subsection{Probiotics}

Probiotics are defined as "live microorganisms which, when administered in adequate amounts as part of food, confer a health benefit on the host"[23]. Probiotics could act at different levels in the immune tolerance network: modulating

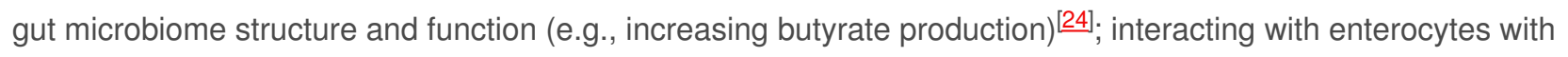
subsequent modulation of nonimmune (gut permeability and mucus thickness) ${ }^{[25}[26]$ and immune tolerogenic mechanisms (stimulation of secretory IgA and $\beta$-defensin production) [27]; and modulation of cytokine response by immune cells[28][29][30][31][32]. Probiotic supplementation represents an interesting option to prevent and treat FA. The most common probiotic bacteria fall into two groups, namely lactobacilli and bifidobacteria.

Recent preclinical studies on probiotic activity against FA were carried out in a murine model of egg allergy. Lactobacillus reuteri AB425917 restored the deteriorated profile of gut microbiota and the imbalance of Th1/Th2, inducing intestinal immune tolerance against ovalbumin-induced allergic response ${ }^{[33]}$. Song et al. isolated and identified Lactobacillus rhamnosus 2016SWU.05.0601, able to restore the immune imbalance of Th1/Th2 and Treg/Th17 in ovalbumin-sensitized mice by modulating gut microbiota, which contributed to the decrease in serum IgE and ovalbumin-lgE levels $3[34$.

In a mouse model of shellfish allergy, oral administration of probiotic strainBifidobacterium infantis 14.518 effectively suppressed tropomyosin-induced allergic response in both preventive and therapeutic strategies. Further results showed that Bifidobacterium infantis 14.518 stimulated DC maturation and CD103+ tolerogenic DC accumulation in gut-associated lymphoid tissue, which subsequently induced Treg cell differentiation aimed at suppressing Th2-biased response. The authors showed that Bifidobacterium infantis 14.518 regulates the alterations of gut microbiota composition. Specifically, the increase of Dorea and decrease of Ralstonia was highly correlated with Th2/Treg ratio and may contribute to alleviating tropomyosin-induced allergic responses $[35]$. 


\section{Encyclopedia}

Preclinical studies were also conducted in murine models of CMA. Neonatal monocolonization of germ-free mice by Lactobacillus caseiBL2 modulated the allergic sensitization to cow's milk proteins.Lactobacillus-casei-colonized mice developed higher casein-specific IgG responses because of casein hydrolysis by Lactobacillus casei into immunogenic peptides ${ }^{[36]}$. Similar results were reported by other authors who observed decreased of concentrations of IgE, IL-4, and IL-13 following administration of Bifidobacterium infantis CGMCC313-2 in BLG-sensitized mice 377 .

Clinical studies have investigated the efficacy of selected probiotic strains against FA. The effect appears to be strainspecific. Among various probiotics, Lactobacillus rhamnosus GG (LGG) has emerged as a bacterial strain able to exert antiallergic actions in humans, especially in CMA. We showed that in CMA children, an extensively hydrolyzed casein formula (EHCF) supplemented with LGG induced higher tolerance rates after 6 and 12 months compared with EHCF alone and other formulas 38$][39]$. At the 3 year follow-up of a pediatric cohort of 220 infants with CMA, further confirmation of a greater rate of oral tolerance acquisition as well as a lower incidence of other allergic manifestations was described after treatment with $E H C F+L G G$ compared with EHCF alone ${ }^{[40]}$. Moreover, we showed that treatment of CMA infants with EHCF+LGG resulted in the enrichment of specific strains of bacteria that are associated with higher fecal butyrate levels ${ }^{[41]}$. The World Allergy Organization guidelines consider the modulation of the immune system using functional foods a promising research hypothesis, as part of efforts to induce a tolerogenic immune environment in the context of CMA. However, the authors concluded that more evidence from randomized controlled trials is needed. They identified further research on probiotic supplementation in CMA treatment as an important area for the development of a stronger evidence base in CMA[42][43].

LGG has also been studied in patients with peanut allergies. In a clinical trial, LGG was administered with peanut oral immunotherapy for 18 months. Subjects receiving the combination treatment had higher rates of desensitization to peanut compared to placebo $(82.1 \% \text { vs. } 3.6 \% \text {, respectively })^{[44]}$. A follow-up study of 48 of the 56 children who participated in this combined probiotic and oral immunotherapy trial showed that treated individuals were more likely to have continued eating peanut compared with those who took a placebo, four years after treatment cessation $(67 \%$ vs. $4 \%, p=0.001$ ); moreover, more participants from the treated group had smaller peanut skin-prick test size and higher peanut slgG4:slgE ratios compared with placebo-treated controls $\frac{[45]}{\text {. }}$

\subsection{Prebiotics}

A prebiotic is now defined as "a substrate that is selectively utilized by host microorganisms conferring a health benefit", including nondigestible compounds, such as oligosaccharides or soluble fermentable fibers that are selectively utilized and promote the growth of beneficial microorganisms and improve health ${ }^{[46]}$. In particular, the galacto-oligosaccharides (GOS)/fructo-oligosaccharides (FOS) combination is the most studied. The mechanisms of action of prebiotics are due to direct and indirect effects. Indirect effects include selective fermentation, increasing populations of resident health-promoting microorganisms of the gut. SCFAs mediate prebiotics' direct beneficial effects at the intestinal and extraintestinal level[47][48]. The supplementation of prebiotics has been proposed as a possible method of intervention in the prevention of allergic disorders $[49]$. However, the vast majority of the systematic reviews and meta-analyses conducted in this area have concluded that although several studies show a positive effect of prebiotics on allergic manifestations, the existing evidence is not sufficient to recommend prebiotic as a routine method for allergy prevention in formula-fed infants $[50 \mid[51]$. Thus, further rigorous studies in this field are required.

\subsection{Postbiotics}

The term postbiotic refers to the use of nonviable cells or cell fractions which, when administered in adequate amounts, confer a health benefit to the host. Additionally, the term postbiotic is also related to soluble components such as SCFAs, vitamins, bacteriocins, organic acids, enzymes, hydrogen peroxide, ethanol, diacetyl, peptides, cellsurface proteins, teichoic acids, peptidoglycan-derived muropeptides, endo- and exopolysaccharides, lactocepins, plasmalogens, polyphosphates, and quorum-sensing molecules produced by live probiotic cells in fermentation processes or synthetically produced in a laboratory [52][53]. The immunomodulatory mechanisms elicited by SCFAs represent one of the strongest connections between diet, gut microbiome, and allergic diseases ${ }^{[\underline{54}]}$. In a human cohort of 3011 -year old children, significant associations were reported between the composition of dietary intake and stool SCFA content, suggesting that diet can be used to modulate microbial production of SCFAs. The authors also investigated the role of SCFAs in allergy prevention and found that the children with the highest levels of butyrate had 


\section{Encyclopedia}

a reduced risk of becoming sensitized to food allergens ${ }^{[55]}$. As we said above, preclinical studies have shown that among SCFAs, butyrate contributes to protection against the development of FA through multiple tolerogenic mechanisms. In human observational studies, butyrate deficiency was observed in allergic children $[56]$, whereas an enrichment of butyrate-producing taxa (Clostridia class and Firmicutes phylum) was observed in children with faster CMA resolution [57]. More recently, Caitet al., using shotgun sequencing, analyzed the fecal microbiomes (at 3 month and 1 year stool samples) of 105 atopic children from the Canadian Healthy Infant Longitudinal Development (CHILD) study to investigate whether bacterial butyrate production in the early-infancy gut is protective against the development of atopic diseases later in life. The authors found that bacteria involved in butyrate production were rather depleted in 3-month-old infants who later developed atopy. Analyzing the gut microbiome function, they also found that 3-monthold infants who later had allergic manifestations lacked genes encoding key enzymes for both carbohydrate breakdown and butyrate production $[58$. We evaluated the direct effects of butyrate on peripheral blood mononuclear cells (PBMCs) from children affected by challenge-proven IgE-mediated CMA. PBMCs were stimulated with BLG in the presence or absence of butyrate. Preliminary results show that butyrate stimulates IL-10 and IFN- $\gamma$ production and decreases the DNA methylation rate of two cytokine genes.

These data suggest the potential of a postbiotic approach, based on the use of SCFAs against FA. However, clinical trials based on SCFA supplementation for FA prevention and treatment have yet to be undertaken. Therefore, there is no current recommendation from any scientific society on the optimal postbiotic administration frequency for the prevention and treatment of FA.

\subsection{Synbiotics}

Synbiotics are a mixture of prebiotics and probiotics that affect the host by improving the survival and implantation of live microbial dietary supplements in the gastrointestinal tract, improving the health of the host $[59]$. Candy et al. $[60]$ designed a study to investigate whether synbiotic ingredients could improve the gut microbiota in infants with non-lgEmediated CMA to achieve a microbial composition close to that seen in healthy, breastfed infants. Infants with suspected non-IgE-mediated CMA were administered the test formula containing the synbiotics, or a control formula without the synbiotics. The test formula was a hypoallergenic, nutritionally complete amino-acid-based formula, including a prebiotic blend of fructo-oligosaccharides and the probiotic strain Bifidobacterium breve M-16V. The control formula was an amino-acid-based formula without synbiotics. The authors concluded that the amino-acid-based formula containing specific synbiotics improved the fecal microbiota of infants with suspected non-lgE-mediated CMA, approximating the composition of the gut microbiota of healthy, breastfed infants.

Interestingly, Bifidobacterium breve M-16V may alter the gut microbiota to alleviate allergy symptoms by IL-33/ST2 signaling. These results indicated that gut microbiota is essential for regulating FA to dietary antigens, and demonstrated that intervention in bacterial community regulation may be therapeutically related to $F A$ [61]

However, although these preliminary data are promising, further studies are needed to evaluate the efficacy of this approach on clinical symptoms.

A planned but not yet recruiting randomized, double-blind clinical trial of children at high risk for allergy will compare partially hydrolyzed infant formula with synbiotics vs. standard infant formula (NCT03067714) for the primary outcome of doctor-diagnosed lgE-mediated allergic manifestations.

\subsection{Fecal Microbiota Transplantation}

Fecal microbiota transplantation represents another approach to shape the gut microbiota in FA patients. The idea behind this strategy is that fecal microbiota transplantation from a healthy donor to a disease recipient can restore gut eubiosis by promoting oral tolerance ${ }^{[62][63]}$. Recently, a human study revealed that fecal microbiota transplantation is able to induce remission of infantile allergic colitis through restoration of gut microbiota diversity [64]. However, the available data in this field remain limited and the relevant scientific work is just beginning. A small Phase I open-label trial to evaluate the safety and efficacy of oral encapsulated fecal microbiota for the treatment of peanut allergy is underway (NCT02960074). 


\section{References}

1. Sicherer, S.H.; Sampson, H.A. Food allergy: A review and update on epidemiology, pathogenesis, diagnosis, prevention, and management. J. Allergy Clin. Immunol. 2018, 141, 41-58.

2. Gupta, R.; Holdford, D.; Bilaver, L.; Dyer, A.; Holl, J.L.; Meltzer, D. The economic impact of childhood food allergy in the United States. JAMA Pediatr. 2013, 167, 1026-1031.

3. Rook, G.A. 99th Dahlem conference on infection, inflammation and chronic inflammatory disorders: Darwinian medicine and the 'hygiene' or 'old friends' hypothesis. Clin. Exp. Immunol. 2010, 160, 70-79.

4. Rook, G.A.; Lowry, C.A.; Raison, C.L. Microbial 'Old Friends', immunoregulation and stress resilience. Evol. Med. Public Health 2013, 2013, 46-64.

5. Hanski, I.; von Hertzen, L.; Fyhrquist, N.; Koskinen, K.; Torppa, K.; Laatikainen, T.; Karisola, P.; Auvinen, P.; Paulin, L.; Mäkelä, M.J.; et al. Environmental biodiversity, human microbiota, and allergy are interrelated. Proc. Natl. Acad. Sci. USA 2012, 109, 8334-8339.

6. Iweala, O.I.; Nagler, C.R. The Microbiome and Food Allergy. Ann. Rev. Immunol. 2019, 37, 377-403.

7. Lee, K.H.; Song, Y.;Wu,W.; Yu, K.; Zhang, G. The gut microbiota, environmental factors, and links to the development of food allergy. Clin. Mol. Allergy 2020, 18, 5.

8. Renz, H.; Allen, K.J.; Sicherer, S.H.; Sampson, H.A.; Lack, G.; Beyer, K.; Oettgen, H.C. Food allergy. Nat. Rev. Dis. Primers 2018, 4, 17098.

9. Lyons, K.E.; Ryan, C.A.; Dempsey, E.M.; Ross, R.P.; Stanton, C. Breast Milk, a Source of Beneficial Microbes and Associated Benefits for Infant Health. Nutrients 2020, 12, 1039.

10. Du Toit, G.; Roberts, G.; Sayre, P.H.; Plaut, M.; Bahnson, H.T.; Mitchell, H.; Radulovic, S.; Chan, S.; Fox, A.; Turcanu, V.; et al. Identifying infants at high risk of peanut allergy: The Learning Early About Peanut Allergy (LEAP) screening study. J. Allergy Clin. Immunol. 2013, 131, 135-143.e1-12.

11. Mitselou, N.; Hallberg, J.; Stephansson, O.; Almqvist, C.; Melén, E.; Ludvigsson, J.F. Cesarean delivery, preterm birth, and risk of food allergy: Nationwide Swedish cohort study of more than 1 million children. J. Allergy Clin. Immunol. 2018, 142, $1510-1514$.

12. Biasucci, G.; Rubini, M.; Riboni, S.; Morelli, L.; Bessi, E.; Retetangos, C. Mode of delivery affects the bacterial community in the newborn gut. Early Hum. Dev. 2010, 86, 13-15.

13. Guibas, G.V.; Moschonis, G.; Xepapadaki, P.; Roumpedaki, E.; Androutsos, O.; Manios, Y.; Papadopoulos, N.G. Conception via in vitro fertilization and delivery by Caesarean section are associated with paediatric asthma incidence. Clin. Exp. Allergy 2013, 43, 1058-1066.

14. Greenwood, C.; Morrow, A.L.; Lagomarcino, A.J.; Altaye, M.; Taft, D.H.; Yu, Z.; Newburg, D.S.; Ward, D.V.; Schibler, K.R. Early empiric antibiotic use in preterm infants is associated with lower bacterial diversity and higher relative abundance of Enterobacter. $\mathrm{J}$. Pediatr. 2014, 165, 23-29.

15. Arboleya, S.; Sánchez, B.; Milani, C.; Duranti, S.; Solís, G.; Fernández, N.; de los Reyes-Gavilán, C.G.; Ventura, M.; Margolles, A.; Gueimonde, M. Intestinal microbiota development in preterm neonates and effect of perinatal antibiotics. J. Pediatr. 2015, 166, 538544.

16. Fouhy, F.; Guinane, C.M.; Hussey, S.; Wall, R.; Ryan, C.A.; Dempsey, E.M.; Murphy, B.; Ross, R.P.; Fitzgerald, G.F.; Stanton, C.; et al. High-throughput sequencing reveals the incomplete, short-term recovery of infant gut microbiota following parenteral antibiotic treatment with ampicillin and gentamicin. Antimicrob. Agents Chemother. 2012, 56, 5811-5820.

17. Biasucci, G. Gut perturbation and probiotics in neonatology. J. Pediatr. Neonat. Ind. Med. 2018, 7, e070202.

18. Silvers, K.M.; Frampton, C.M.;Wickens, K.; Pattemore, P.K.; Ingham, T.; Fishwick, D.; Crane, J.; Town, G.I.; Epton, M.J.; New Zealand Asthma and Allergy Cohort Study Group. Breastfeeding protects against current asthma up to 6 years of age. J. Pediatr. 2012, 160, 991-996.

19. Wu, G.D.; Chen, J.; Hoffmann, C.; Bittinger, K.; Chen, Y.Y.; Keilbaugh, S.A.; Bewtra, M.; Knights, D.; Walters, W.A.; Knight, R.; et al. Linking long-term dietary patterns with gut microbial enterotypes. Science 2011, 334, 105-108.

20. Trompette, A.; Gollwitzer, E.S.; Yadava, K.; Sichelstiel, A.K.; Sprenger, N.; Ngom-Bru, C.; Blanchard, C.; Junt, T.; Nicod, L.P.; Harris, N.L.; et al. Gut microbiota metabolism of dietary fiber influences allergic airway disease and hematopoiesis. Nat. Med. 2014, 20, $159-166$.

21. Jordakieva, G.; Kundi, M.; Untersmayr, E.; Pali-Schöll, I.; Reichardt, B.; Jensen-Jarolim, E. Country-wide medical records infer increased allergy risk of gastric acid inhibition. Nat. Commun. 2019, 10, 3298.

22. Schöll, I.; Ackermann, U.; Ozdemir, C.; Blümer, N.; Dicke, T.; Sel, S.; Sel, S.; Wegmann, M.; Szalai, K.; Knittelfelder, R.; et al. Antiulcer treatment during pregnancy induces food allergy in mouse mothers and a Th2-bias in their offspring. FASEB J. 2007, 21, 12641270.

23. Hill, C.; Guarner, F.; Reid, G.; Gibson, G.R.; Merenstein, D.J.; Pot, B.; Morelli, L.; Berni Canani, R.; Flint, H.J.; Salminen, S.; et al. The International scientific association for probiotics and prebiotics consensus statement on the scope and appropriate use of the term probiotics. Nat. Rev. Gastrol. Hepat. 2014, 11, 506-514.

24. Berni Canani, R.; Sangwan, N.; Stefka, A.; Nocerino, R.; Paparo, L.; Aitoro, R.; Calignano, A.; Khan, A.A.; Gilbert, J.A.; Nagler, C.R. Lactobacillus rhamnosus GG-supplemented formula expands butyrate-producing bacterial strains in food allergic infants. ISME J. 2016, 10, 742-750.

25. Paparo, L.; Aitoro, R.; Nocerino, R.; Fierro, C.; Bruno, C.; Berni Canani, R. Direct e_ects of fermented cow's milk product with 
Lactobacillus paracasei CBA L74 on human enterocytes. Benef. Microbes 2018, 9, 165-172.

26. Tulyeu, J.; Kumagai, H.; Jimbo, E.; Watanabe, S.; Yokoyama, K.; Cui, L.; Osaka, H.; Mieno, M.; Yamagata, T. Probiotics Prevents Sensitization to Oral Antigen and Subsequent Increases in Intestinal Tight Junction Permeability in Juvenile-Young Adult Rats. Microorganisms 2019, 7, 463.

27. Hardy, H.; Harris, J.; Lyon, E.; Beal, J.; Foey, A.D. Probiotics, Prebiotics and Immunomodulation of gut mucosal defences: Homeostatis and immunopathology. Nutrients 2013, 5, 1869-1912.

28. Furusawa, Y.; Obata, Y. Commensal microbe-derived butyrate induces differentiation of colonic regulatory T cells. Nature 2013, 504, 446-450.

29. Di Costanzo, M.; Paparo, L.; Aitoro, R.; Cosenza, L.; Nocerino, R.; Cozzolino, T.; Pezzella, V.; Vallone, G.; Berni Canani, R. Potential Beneficial Effects of Butyrate against Food Allergy. In Butyrate: Food Sources, Functions and Health Benefits; Li, C.-J., Ed.; Biochemistry Research Trends: New York, NY, USA, 2014; pp. 81-90.

30. Torii, A.; Torii, S.; Fujiwara, S.; Tanaka, H.; Inagaki, N.; Nagai, H. Lactobacillus acidophilus strain L-92 regulates the production of Th1 cytokine as well as Th2 cytokines. Allergol. Int. 2007, 56, 293-301.

31. Niers, L.E.; Timmerman, H.M.; Rijkers, G.T.; van Bleek, G.M.; van Uden, N.O.; Knol, E.F.; Kapsenberg, M.L.; Kimpen, J.L.; Hoekstra, M.O. Identification of strong interleukin?10 inducinglactic acid bacteria which down-regulate T helper type 2 cytokines. Clin. Exp. Allergy 2005, 35, 1481-1489.

32. Takahashi, N.; Kitazawa, H.; Iwabuchi, N.; Xiao, J.Z.; Miyaji, K.; Iwatsuki, K.; Saito, T. Oral administration of an immunostimulatory DNA sequence from Bifidobacterium longum improves Th1/Th2 balance in a murine model. Biosci. Biotechnol. Biochem. 2006, 70, 2013-2017.

33. Huang, C.H.; Lin, Y.C.; Jan, T.R. Lactobacillus reuteri induces intestinal immune tolerance against food allergy in mice. J. Funct. Foods 2017, 31, 44-51.

34. Song, J.; Li, Y.; Li, J.; Wang, H.; Zhang, Y.; Suo, H. Lactobacillus rhamnosus 2016SWU.05.0601 regulates immune balance in ovalbumin-sensitized mice by modulating the immune-related transcription factors expression and gut microbiota. J. Sci. Food Agric. 2020.

35. Fu, L.; Song, J.; Wang, C.; Fu, S.; Wang, Y. Bifidobacterium infantis Potentially Alleviates Shrimp Tropomyosin-Induced Allergy by Tolerogenic Dendritic Cell-Dependent Induction of Regulatory T Cells and Alterations in Gut Microbiota. Front. Immunol. 2017, 8, 1536.

36. Maiga, M.A.; Morin, S.; Bernard, H.; Rabot, S.; Adel-Patient, K.; Hazebrouck, S. Neonatal mono-colonization of germ-free mice with Lactobacillus casei enhances casein immunogenicity after oral sensitization to cow's milk. Mol. Nutr. Food Res. $2017,61,1600862$.

37. Liu, M.Y.; Yang, Z.Y.; Dai, W.K.; Huang, J.Q.; Li, Y.H.; Zhang, J.; Qiu, C.Z.; Wei, C.; Zhou, Q.; Sun, X.; et al. Protective effect of Bifidobacterium infantis CGMCC313-2 on ovalbumin-induced airway asthma and b-lactoglobulin induced intestinal food allergy mouse models. World J. Gastroenterol. 2017, 23, 2149-2158.

38. Berni Canani, R.; Nocerino, R.; Terrin, G.; Coruzzo, A.; Cosenza, L.; Leone, L.; Troncone, R. Effect of Lactobacillus GG on tolerance acquisition in infants with cow's milk allergy a randomized trial. J. Allergy Clin. Immunol. 2012, 129, 580-582.

39. Berni Canani, R.; Nocerino, R.; Terrin, G.; Frediani, T.; Lucarelli, S.; Cosenza, L.; Passariello, A.; Leone, L.; Granata, V.; Di Costanzo, M.; et al. Formula selection for management of children with cow milk allergy influences the rate of acquisition of tolerance: A prospective multicenter study. J. Pediatr. 2013, 163, 771-777.

40. Berni Canani, R.; Di Costanzo, M.; Bedogni, G.; Amoroso, A.; Cosenza, L.; Di Scala, C.; Granata, V.; Nocerino, R. Extensively hydrolyzed casein formula containing Lactobacillus rhamnosus GG reduces the occurrence of other allergic manifestation sin children with cow's milk allergy: 3-year randomized controlled trial. J. Allergy Clin. Immunol. 2017, 139, 1906-1913.

41. Russell, S.L.; Gold, M.J.; Hartmann, M.;Willing, B.P.; Thorson, L.; Wlodarska, M.; Gill, N.; Blanchet, M.R.; Mohn, W.W.; McNagny, K.M.; et al. Early life antibiotic-driven changes in microbiota enhance susceptibility to allergic asthma. EMBO Rep. 2012, 13, 440447.

42. Fox, A.; Bird, J.A.; Fiocchi, A.; Knol, J.; Meyer, R.; Salminen, S.; Sitang, G.; Szajewska, H.; Papadopoulos, N. The potential for pre-, pro- and synbiotics in the management of infants at risk of cow's milk allergy or with cow's milk allergy: An exploration of the rationale, available evidence and remaining questions. World Allergy Organ. J. 2019, 12, 100034.

43. Fiocchi, A.; Brozek, J.; Schunemann, H.; Bahna, S.L.; von Berg, A.; Beyer, K.; Bozzola, M.; Bradsher, J.; Compalati, E.; Ebisawa, M.; et al. World allergy organization (WAO) diagnosis and rationale for action against cow's milk allergy (DRACMA) guidelines. World Allergy Organ. J. 2010, 21, 57-161.

44. Tang, M.L.; Ponsonby, A.L.; Orsini, F.; Tey, D.; Robinson, M.; Su, E.L.; Licciardi, P.; Burks, W.; Donath, S. Administration of a probiotic with peanut oral immunotherapy: A randomized trial. J. Allergy Clin. Immunol. 2015, 135, 737-744.e8.

45. Hsiao, K.C.; Ponsonby, A.L.; Axelrad, C.; Pitkin, S.; Tang, M.; PPOIT Study Team. Long-term clinical and immunological effects of probiotic and peanut oral immunotherapy after treatment cessation: 4-year follow-up of a randomised, double-blind, placebocontrolled trial. Lancet Child. Adolesc. Health 2017, 1, 97-105.

46. Gibson, G.R.; Hutkins, R.; Sanders, M.E.; Prescott, S.L.; Reimer, R.A.; Salminen, S.J.; Scott, K.; Stanton, C.; Swanson, K.S.; Cani, P.; et al. Expert consensus document: The International Scientific Association for Probiotics and Prebiotics (ISAPP) consensus statement on the definition and scope of prebiotics. Nat. Rev. Gastroenterol. Hepatol. 2017, 14, 491-502. 
47. Berni Canani, R.; Di Costanzo, M.; Leone, L.; Pedata, M.; Meli, R.; Calignano, A. Potential beneficial effects of butyrate in intestinal and extraintestinal diseases. World J. Gastroenterol. 2011, 17, 1519-1528.

48. Miqdady, M.; Al Mistarihi, J.; Azaz, A.; Rawat, D. Prebiotics in the Infant Microbiome: The Past, Present, and Future. Pediatr Gastroenterol. Hepatol. Nutr. 2020, 23, 1-14.

49. Tang, M.L.; Lahtinen, S.J.; Boyle, R.J. Probiotics and prebiotics: Clinical effects in allergic disease. Curr. Opin. Pediatr. 2010, 22, 626-634.

50. Osborn, D.A.; Sinn, J.K. Prebiotics in infants for prevention of allergy. Cochrane Database Syst. Rev. 2013, 3, CD006474.

51. Braegger, C.; Chmielewska, A.; Decsi, T.; Kolacek, S.; Mihatsch, W.; Moreno, L.; Piescik, M.; Puntis, J.; Shamir, R.; Szajewska, H.; et al. Supplementation of infant formula with probiotics and/or prebiotics: A systematic review and comment by the ESPGHAN committee on nutrition. J. Pediatr Gastroenterol. Nutr. 2011, 52, 238-250.

52. Homayouni Rad, A.; Aghebati Maleki, L.; Samadi Kafil, H.; Abbasi, A. Postbiotics: A novel strategy in food allergy treatment. Crit. Rev. Food Sci. Nutr. 2020, 1-8.

53. Rad, A.H.; Maleki, L.A.; Kafil, H.S.; Zavoshti, H.F.; Abbasi, A. Postbiotics as novel health-promoting ingredients in functional foods. Health Promot. Perspect. 2020, 10, 3-4.

54. McKenzie, C.; Tan, J.; Macia, L.; Mackay, C.R. The nutrition-gut microbiome-physiology axis and allergic diseases. Immunol. Rev. 2017, 278, 277-295.

55. Roduit, C.; Frei, R.; Ferstl, R.; Loeliger, S.; Westermann, P.; Rhyner, C.; Schiavi, E.; Barcik, W.; Rodriguez-Perez, N.; Wawrzyniak, M.; et al. High levels of butyrate and propionate in early life are associated with protection against atopy. Allergy $2019,74,799-809$.

56. Sandin, A.; Bråbäck, L.; Norin, E.; Björkstén, B. Faecal short chain fatty acid pattern and allergy in early childhood. Acta Paediatr. 2009, 98, 823-827.

57. Bunyavanich, S.; Shen, N.; Grishin, A.; Wood, R.; Burks, W.; Dawson, P.; Jones, S.M.; Leung, D.; Sampson, H.; Sicherer, S.; et al. Early-life gut microbiome composition and milk allergy resolution. J. Allergy Clin. Immunol. 2016, 138, 1122-1130.

58. Cait, A.; Cardenas, E.; Dimitriu, P.A.; Amenyogbe, N.; Dai, D.; Cait, J.; Sbihi, H.; Stiemsma, L.; Subbarao, P.; Mandhane, P.J.; et al. Reduced genetic potential for butyrate fermentation in the gut microbiome of infants who develop allergic sensitization. J. Allergy Clin. Immunol. 2019, 144, 1438-1647.

59. Markowiak, P.; Slizewska, K. E_ects of probiotics, prebiotics, and synbiotics on human health. Nutrients $2017,9,1021$.

60. Candy, D.C.A.; Van Ampting, M.T.J.; Oude Nijhuis, M.M.; Wopereis, H.; Butt, A.M.; Peroni, D.G.; Vandenplas, Y.; Fox, A.T.; Shah, N.; West, C.E.; et al. A synbiotic-containing amino-acid-based formula improves gut microbiota in non-IgE-mediated allergic infants. Pediatr. Res. 2018, 83, 677-686.

61. Li, N.; Yu, Y.; Chen, X.; Gao, S.; Zhang, Q.; Xu, C. Bifidobacterium breve M-16V alters the gut microbiota to alleviate OVA-induced food allergy through IL-33/ST2 signal pathway. J. Cell. Physiol. 2020.

62. Borody, T.J.; Khoruts, A. Fecal microbiota transplantation and emerging applications. Nat. Rev. Gastroenterol. Hepatol. 2011, 9, 8896.

63. Gupta, S.; Allen-Vercoe, E.; Petrof, E.O. Fecalmicrobiota transplantation: In perspective. Ther. Adv. Gastroenterol. 2016, 9, 229239.

64. Liu, S.-X.; Li, Y.-H.; Dai, W.-K.; Li, X.S.; Qiu, C.Z.; Ruan, M.L.; Zou, B.; Dong, C.; Liu, Y.H.; He, J.Y.; et al. Fecal microbiota transplantation induces remission of infantile allergic colitis through gut microbiota re-establishment. World J. Gastroenterol. 2017, 23, 8570-8581.

\section{Keywords}

gut microbiome; food allergy; dysbiosis

(C) 2020 by the author(s). Distribute under a Creative Commans CC BY license 